\title{
ON NAIVE TAYlor MOdel INTEgRATION METHOD
}

\author{
Shawki A. M. Abbas*
}

Date of acceptance 16/9/2008

\begin{abstract}
:
Interval methods for verified integration of initial value problems (IVPs) for ODEs have been used for more than 40 years. For many classes of IVPs, these methods have the ability to compute guaranteed error bounds for the flow of an ODE, where traditional methods provide only approximations to a solution. Overestimation, however, is a potential drawback of verified methods. For some problems, the computed error bounds become overly pessimistic, or integration even breaks down. The dependency problem and the wrapping effect are particular sources of overestimations in interval computations.
\end{abstract}

Berz (see [1]) and his co-workers have developed Taylor model methods, which extend interval arithmetic with symbolic computations. The latter is an effective tool for reducing both the dependency problem and the wrapping effect. By construction, Taylor model methods appear particularly suitable for integrating nonlinear ODEs. In this paper, we analyze Taylor model based integration of ODEs and compare Taylor model with traditional enclosure methods for IVPs for ODEs.

More advanced Taylor model integration methods are discussed in the algorithm (1). For clarity, we summarize the major steps of the naive Taylor model method as algorithm 1 .

Key Words: Taylor model methods, verified integration, ODEs, IVPs.

\section{Introduction}

Taylor model methods use multivariate polynomials in the initial values plus a small interval remainder term to represent the flow of an IVP. Thus, it is possible to work with nonlinear boundary curves. Including non-convex enclosure sets for crescentshaped or twisted flows. For non linear ODEs, this increased flexibility to admissible boundary curves is an intrinsic advantage of Taylor model methods over traditional interval methods, making Taylor methods very effective in some cases in reducing the wrapping effect.

We refer to recent paper of Makino and Berz[2] for the general description of Taylor model methods for ODEs. Our intention here is to explain the fundamental difference between interval methods and Taylor model methods with a simple nonlinear examples.

\section{Shrink Wrapping and Preconditioning}

For successful integration over long time spans, sophisticated treatment of the interval terms is required. For this purpose, Berz and Makino see [3] invented two schemes which they call shrink wrapping and preconditioning. Shrink wrapping is a method to absorb the interval reminder term into the symbolic part of the Taylor model. From a geometric viewpoint, it resembles the parallelepiped method. Shrink wrapping use the same linear map as

* Department of Mathematics- College of Science- University of Baghdad 
the parallelepiped at maintaining a small condition number for the shrink wrapping map. Thus it stabilizes the integration process, like the QR interval method does.

For clarity of the presentation, we describe the shrink wrapping and preconditioning for the special case of linear autonomous ODEs. The generalization to nonlinear ODEs are straightforward. We refer to [4] for more details.

\section{Taylor Model Methods for Linear ODEs}

For a linear ODE, the flow of an interval IVP is a parallelepiped for all time, so Taylor models seems to have no obvious advantage over interval methods. On the other hand, if Taylor model methods failed on linear ODEs, they would probably not be effective for nonlinear ODEs. The purpose of this selection is to show that they can be as good as interval methods for linear ODEs. ODE:

Consider the linear autonomous

$\mathrm{U}^{\prime}=\mathrm{BU}$

$\mathrm{U}(0)=\mathrm{U}_{0}$

Where $\mathrm{B}$ is a given real matrix, $\mathbf{x}$ is a given interval vector, and $\mathrm{U}_{0}=\operatorname{Pn}(\mathrm{x}), \mathrm{x} \in \mathrm{x}$, is a Taylor model vector with zero remainder interval describing the initial set. $\mathrm{x}$ is used to denote the vector of the step space variables. Assume that the enclosure step in the Taylor model method is feasible with some constant step size $\mathrm{h}>0$ and some order $\mathrm{n} \in \mathrm{N}$.

\section{Naive Taylor Model method}

In the first integration step, Picard integration of order $\mathrm{n}$ is used to compute the multivariate Taylor polynomial:

$$
\mathrm{U}_{1, \mathrm{n}}:=\mathrm{P}_{\mathrm{n}}(\mathrm{tB}) \mathrm{P}_{\mathrm{n}}(\mathrm{x})
$$

$$
\text { Where } \quad P_{n}(t B):=\sum_{k=0}^{n} \frac{(t B)^{k}}{k !}
$$

introducing $\mathrm{T}:=\mathrm{P}_{\mathrm{n}}(\mathrm{hB})$, the verification step consists of finding an interval $i_{1}$ such that:

$$
\begin{gathered}
P_{n}(x)+\int_{0}^{h} B\left(P_{n}(T B) P_{n}(x)+i_{1}\right) d T \subseteq \\
P_{n}(h B) P_{n}(x)+i_{1}=T P_{n}(x)+i_{1}
\end{gathered}
$$

holds for all $\mathrm{x} \in \mathrm{x}$ (see for example [2, ch.6]. At $t_{1}=h$, the flow of the IVP (1) is then enclosed by the Taylor model $\mathrm{U} 1:=\mathrm{TPn}(\mathrm{x})+\mathrm{i}_{1}$.

Subsequent integration steps are performed in the same manner, but with a slight modification in the verification step. In the $\mathrm{j}^{\text {th }}$ integration step, $\mathrm{j} \geq 2, \mathrm{i}_{\mathrm{j}}$ is sought such that the inclusion

$T^{j-1} P_{n}(x)+i_{j-1}+\int_{0}^{h} B\left(P_{n}(T B) T^{j-1} P_{n}(x)+i_{j}\right) d T \subseteq T^{j} P_{n}(x)+i_{j}$

Is fulfilled for all $\mathrm{x} \in \mathrm{x}$ letting:

$\mathrm{U}_{\mathrm{j}}:=\mathrm{Tu}_{\mathrm{j}-1}+\mathrm{i}_{\mathrm{j}}, \mathrm{j}=1,2$,

The naive Taylor model method for (1) consists of the iteration

$$
\mathrm{U}_{\mathrm{j}}=\mathrm{T}^{\mathrm{j}} \mathrm{U}_{0}+\sum_{\mathrm{k}=1}^{\mathrm{j}}\left(\mathrm{T}_{0}\right)^{\mathrm{j}-\mathrm{k}} \mathrm{i}_{\mathrm{k}}, \quad \mathrm{j}=1,2, \ldots
$$

Where $\left(\mathrm{T}_{0}\right)^{0} \mathbf{x}=\mathbf{x},\left(\mathrm{T}_{0}\right)^{\mathrm{k}} \mathbf{x}=\mathrm{T}$. $\left(\left(\mathrm{T}_{0}\right)^{\mathrm{k}+1} \mathbf{x}\right), \mathrm{k} \in \mathrm{N}$. A part from the different computation of the remainder interval, for IVP(1). The naive Taylor model method (5) coincides with the direct interval method that occurs in [6]. Hence the naive Taylor model method (5) has the same divergence property as the direct interval method, 
for which it was shown in [6] that after j steps we have:

$$
w\left(\left(T_{0}\right)^{j-1} i_{1}\right)=|T|^{j-1} w\left(i_{1}\right) .
$$

(for $\mathrm{A}=\left(\mathrm{a}_{\mathrm{ij}}\right)$, we denoted by $|\mathrm{A}|$ the matrix with components $\left.\left|a_{i j}\right|\right)$. The key point here is that the spectral radius of $|\mathrm{T}|^{j-1}$ may be much larger than spectral radius of $\mathrm{T}^{\mathrm{j}-1}$, which describes the natural error growth of a point method. If this is the case, the error bounds for the naive Taylor model method may be much larger than the true error.

\section{Naive Taylor Model Method with Shrink Wrapping}

Berz and Makina [2] defined shrink wrapping as a method for absorbing the interval part of the Taylor model into the polynomial part by modifying the polynomial coefficients. The set defined by the sum of the giving polynomial and interval is wrapped by a set defined by a pure polynomial. The new set may be larger than the initial set, but it is less prone to the dependency problem and to the wrapping effect in succeeding calculations.

In the verified integration of ODEs, shrink wrapping is usually applied to the Taylor model enclosures of the flow at the grid points, before continuing the integration. In practical computations, shrink wrapping is performed when the size of the interval remainder term exceeds some heuristically chosen bound. After shrink wrapping, the initial set of the subsequent integration step is purely symbolic, which removes the dependency problem and simplifies the verification step. The success of Taylor model based integration method depends on the successful reduction of the excess introduced in the shrink wrapping process.
The process of applying shrinks wrapping to a Taylor model vector:

$\mathrm{U}:=\mathrm{P}(\mathrm{x})+\mathrm{i} . \mathrm{x} \in \mathrm{x}$.

Is described in [11]. Here we only outline its four basic steps.

First, let $\widetilde{U}$ denoted the Taylor model that is obtain when the constant part of $\mathrm{P}$ is removed.

Second, multiply $\widetilde{U}$ by the inverse of the matrix associated with its linear part and obtain the Taylor model $\tilde{\mathrm{U}}$.

Third, estimate the nonlinear part of $\widetilde{U}$, its Jacobian, and the interval term of $\tilde{U}$, to obtain the shrink wrap factor $\mathrm{q} \geq 1$.

Forth, multiply the polynomial part of $\tilde{U}$ with $q$ and add the constant part of $U$.

We illustrate shrink wrapping with the following nonlinear example. For clarity, we use two scalar Taylor models $\mathrm{U}$ and $\mathrm{V}$ instead of a Taylor model vector, the symbolic variables are denoted by $\mathrm{a}$ and $\mathrm{b}$ (instead of the vector $\mathrm{x}$ ).

Example: Absorption of the interval part into the symbolic part of a Taylor model. We consider the Taylor model vector $(\mathrm{U}, \mathrm{v})^{\mathrm{T}}$, where:

$$
\begin{aligned}
& \mathrm{u}(\mathrm{a}, \mathrm{b}):=2+4 \mathrm{a}+\frac{1}{2} \mathrm{a}^{2}+[-0.2,0.2] \\
& \mathrm{V}(\mathrm{a}, \mathrm{b}):=1+3 \mathrm{~b}+\mathrm{ab}+[-0.1,0.1] \\
& \text { Where } \mathrm{a}, \mathrm{b} \in[-1,1] \ldots \ldots \ldots .(8)
\end{aligned}
$$

The set defined in (8) is shown in fig 1 . Following the above outline, we obtain:

$$
\begin{aligned}
& \widetilde{U}(a, b):=4 a+\frac{1}{2} a^{2}+[-0.2,0.2] \\
& \widetilde{V}(a, b):=3 b+a b+[-0.1,0.1] \ldots
\end{aligned}
$$

The matrix associated with the linear part of the Taylor model (9) is 
$C:=\left(\begin{array}{ll}4 & 0 \\ 0 & 3\end{array}\right)$ multiplying (9) by $C^{-1}$, yields:

$\widetilde{\mathrm{U}}(\mathrm{a}, \mathrm{b})=\mathrm{a}+\frac{1}{8} \mathrm{a}^{2}+[-0.05,0.05]$

$\tilde{\mathrm{V}}(\mathrm{a}, \mathrm{b})=\mathrm{b}+\frac{1}{3} \mathrm{ab}+[-0.034,0.034]$

Estimating the nonlinear part and the interval terms as described in [2], and compute the numbers $\mathrm{s}, \mathrm{t}$, and $\mathrm{d}$ that satisfying

$\mathrm{s} \geq\left|\frac{1}{8} \mathrm{a}^{2}\right|, \mathrm{s} \geq\left|\frac{1}{3} \mathrm{ab}\right|$ for all $\mathrm{a}, \mathrm{b} \in[-1,1]$,

$\mathrm{t} \geq\left|\frac{1}{4} \mathrm{a}\right|, \mathrm{t} \geq\left|\frac{1}{3} \mathrm{~b}\right|, \mathrm{t} \geq\left|\frac{1}{3} \mathrm{a}\right|$, for all $\mathrm{a}, \mathrm{b} \in[-1,1]$

$d \geq 0.05, d \geq 0.034$
These conditions are fulfilled for $\mathrm{s}=\mathrm{t}=1 / 3$ and $\mathrm{d}=0.05$, from which one can deduce the shrink warp factor.

$q=1+d \cdot \frac{1}{(1-t)(1-s)}=\frac{89}{80}$

The final Taylor model after shrink wrapping is:

$\mathrm{u}_{\mathrm{sw}}(\mathrm{a}, \mathrm{b})=2+\frac{89}{80} \mathrm{a}+\frac{89}{160} \mathrm{a}^{2}$,

$\mathrm{V}_{\text {sw }}(\mathrm{a}, \mathrm{b})=1+\frac{287}{80} \mathrm{~b}+\frac{89}{80} \mathrm{ab}$ by (11).

As Figure 1 show, the set defined

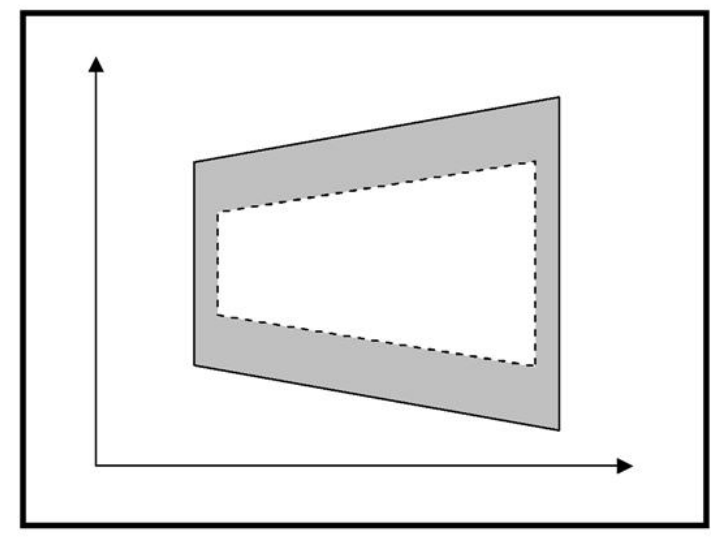

Fig. 1: Sets of Taylor models before (Eq. (8)) and after shrink wrapping (Eq. (11)). The dotted line is the boundary of the set that is described by the polynomial of the original Taylor model. The white area is the set described by the original Taylor model, inchuding the interval term. The excess area introduced by shrink wrapping is shaded in grey.

Applying shrink wrapping in the linear model problem (1) is rather simple. For simplicity, let us assume that the shrink wrapping is performed in every integration step. Then we must compute [11] $\mathrm{q}_{\mathrm{j}}:=1+\mathrm{d}_{\mathrm{j}} / 2$, where $\mathrm{d}_{\mathrm{j}}:=\left\|\mathrm{w}\left(\left(\mathrm{T}^{\mathrm{j}}\right)^{-1} \mathrm{i}_{\mathrm{j}}\right)\right\|_{\infty}$ if $\mathrm{T}$ is sufficiently well-condition, and if the interval terms are sufficiently small, then the factor $d_{j}$ are almost zero, and shrink wrapping is feasible for many integration steps. 
The naive Taylor model method with shrink wrapping resembles the parallelepiped method. By multiplying the non-constant coefficients of the Taylor polynomial, for linear autonomous ODEs the interval term is absorbed as in the parallelepiped method. While $\mathrm{T}^{\mathrm{j}}$ is well-conditioned, $\mathrm{d}_{\mathrm{j}}$ is small, and so is the excess area. On the other hand, $\mathrm{q}_{j}$ (and the excess area) becomes large if $\mathrm{T}^{\mathrm{j}}$ become ill conditioned, which is eventually the case if $T$ has eigenvalues of different magnitude. In this case the integration breaks down due to the growth of the Taylor polynomial coefficients.

\section{Algorithm 1 (Naive Taylor Model Method)}

Let the initial set be given as a Taylor model vector in $\mathrm{m}$ space variables.

For $\mathrm{j}=0,1, \ldots, \mathrm{J}_{\max -1}$ :

1. Compute the Taylor polynomial $P_{n}$ (of dimension $\mathrm{m}$ in $\mathrm{m}+1$ variables) of the solution of the $\mathrm{j}+1^{\text {st }}$ time step, using Picard iteration.

2. Compute a remainder interval vector $\mathrm{i}$, using $\mathrm{S}$ chander's fixed point theorem (via interval iteration based on Picard iteration).

3. Evaluate $\widetilde{\mathrm{u}}=\mathrm{P}_{\mathrm{n}}+\mathrm{i}$, the resulting $\mathrm{m}$ dimensional Taylor model u which contains the flow of the IVP and serves as initial set for the next time step.

\section{Quadratic Model Problem} problem

Consider the quadratic model

$$
\mathrm{u}^{\prime}=\mathrm{v}_{1} \quad \mathrm{u}(0) \in[0.95,1.05],
$$$$
v^{\prime}=u^{2} \quad v(0) \in[-1.05,-0.95] \text {, }
$$

Where the differentiation is with respect to $t$. In an interval method, one would use interval initial values $\mathrm{u}_{0}=$ $[0.95,1.05]$ and $v_{0}=[-1.05,-0.95]$. In the Taylor model method, the initial set is described by parameters, which we call $a$ and $b$, and which we choose on the interval $[-0.05,0.05]$. The initial conditions of $\operatorname{IVP}(11)$ at $t=t_{0}$ are thus given by:

$$
\begin{array}{ll}
\mathrm{U}_{0}(\mathrm{a}, \mathrm{b}):=1+\mathrm{a} & \mathrm{a} \in \mathrm{a}:=[-0.05,0.05], \\
\mathrm{V}_{0}(\mathrm{a}, \mathrm{b}):=-1+\mathrm{b} & \mathrm{b} \in \mathrm{b}:=[-0.05,0.05] .
\end{array}
$$

For illustration, we use order $\mathrm{n}=$ 3 and step size $\mathrm{h}=0.1$ in the Taylor model integration of (11). All numbers are displayed here rounded to six decimal digits. In each integration step, the multivariate Taylor series (with respect to $t, a$ and $b$ ) of the solution of (11) is employed. The third-order Taylor polynomial serves as an approximate solution. The truncation error of the series is enclosed by a suitable reminder interval

The first integration step consists of integrating the IVP

$$
\begin{array}{ll}
\mathrm{u}^{\prime}=\mathrm{v}, & \mathrm{u}(0)=1+\mathrm{a} \\
\mathrm{v}^{\prime}=\mathrm{v}^{2}, & \mathrm{v}(0)=-1+\mathrm{b}
\end{array}
$$

3)

For $\mathrm{o} \leq \mathrm{t} \leq \mathrm{h}$. we use the Picard iteration to calculate a multivariate Taylor polynomial approximation of the solution to (12) using the initial approximations

$\mathrm{u}^{(0)}(\mathrm{T}, \mathrm{a}, \mathrm{b})=1+\mathrm{a}, \mathrm{v}^{(0)}(\mathrm{T}, \mathrm{a}, \mathrm{b})=-1+$ $\mathrm{b}$ ( $\mathrm{T}$ is time), the first step of the Picard iteration yields:

$$
\begin{gathered}
U^{(\mathrm{l})}(T, a, b)=U_{0}(a, b)+\int_{0}^{T} V^{(0)}(s, a b) d s=+a-T+b T \\
\left.V^{T}(T, a, b)=V_{0}(a, b)+\int_{0}^{(0)}(s, a b)\right)^{2} d s=-1+b+T+2 a T+a^{2} T
\end{gathered}
$$

After two more Picard iterations (and omitting the higher order terms), we obtain the third order Taylor polynomials.

$U^{(3)}(T, a, b)=1+a-T+b T+\frac{1}{2} T^{2}+a T^{2}-\frac{1}{3} T^{3}$ 


$$
\begin{aligned}
& V^{(3)}(T, a, b)=-1+b+T+2 a T \\
& +a^{2} T-T^{2}+b T^{2}+\frac{2}{3} T^{3}
\end{aligned} .
$$

As multivariate approximations to the solution of (12). For verified enclosure of the flow, the Taylor polynomials have to be furnished with suitable reminder bounds. Their derivation is based on fixed point iteration [12]. Intervals $\mathrm{i}_{0}$ and $\mathrm{j}_{0}$ are sought such that the inclusions:

$$
\begin{aligned}
& \mathrm{U}_{0}+\int_{0}^{\mathrm{T}}\left(\mathrm{V}^{(3)}(\mathrm{s}, \mathrm{a}, \mathrm{b})+\mathrm{j}_{0}\right) \mathrm{ds} \subseteq \mathrm{U}^{(3)}(\mathrm{T}, \mathrm{a}, \mathrm{b})+\mathrm{i}_{0} \\
& \mathrm{~V}_{0}+\int_{0}^{\mathrm{T}}\left(\mathrm{U}^{(3)}(\mathrm{s}, \mathrm{a}, \mathrm{b})+\mathrm{i}_{0}\right)^{2} \mathrm{ds} \subseteq \mathrm{V}^{(3)}(\mathrm{T}, \mathrm{a}, \mathrm{b})+\mathrm{j}_{0}
\end{aligned}
$$

Simultaneously hold for all $\mathrm{a} \in$ $\mathrm{a}$, for all $\mathrm{b} \in \mathrm{b}$, and for all $\mathrm{T} \in[0,0.1]$. For details of this computation of the reminder interval, refer to [12]. In my example, these inclusions are fulfilled.

Example 2: For $\mathrm{i}_{0}=[-5.09307 \mathrm{E}-$ $5,7.86167 \mathrm{E}-5]$ and $\mathrm{j}_{0}=[-1.75707 \mathrm{E}-4$, 1.60933E-4] An enclosure of the flow of the $\operatorname{IVP}(12)$ for $t \in[0,0.1]$ is given by Taylor models:

$\widetilde{\mathrm{U}}(\mathrm{T}, \mathrm{a}, \mathrm{b}):=1+\mathrm{a}-\mathrm{T}+\mathrm{bT}+\frac{1}{2} \mathrm{~T}^{2}+\mathrm{aT}^{2}-\frac{1}{3} \mathrm{~T}^{\hat{3}}+\mathrm{i}_{0}$, $\widetilde{V}_{1}(T, a, b)=-1+b+T+2 a T+a^{2} T-T^{2}-a T^{3}+b T^{2}+\frac{2}{3} T^{\hat{3}}+$

Where $\mathrm{a}, \mathrm{b} \in[-0.05,0.05], \mathrm{T} \in$ $[0,0.1]$, and $\mathrm{t}=\mathrm{T}$.

Evaluating $\widetilde{\mathrm{U}}_{1}$ and $\hat{\mathrm{V}}_{1}$ at $\mathrm{T}=\mathrm{h}=$ 0.1 , we obtain the enclosure of the flow at $\mathrm{t}_{1}=0.1$ (Taylor models of order at most 2 in the space variables):

$$
\begin{aligned}
& u_{1}(a, b):=\tilde{u}_{1}(0.1, a, b)= \\
& 0.904667+1.01 a+0.1 b+i_{0},
\end{aligned}
$$

$$
\begin{aligned}
& v_{1}(a, b):=\widetilde{v}_{1}(0.1, a, b)=-0.909333 \\
& +0.19 a+1.01 b+0.1 a^{2}+j_{0}
\end{aligned}
$$

Which is the initial set for the second integration step. The latter is performed with a slight modification. we do not use the interval remainder terms in $\mathrm{u}_{1}$ and $\mathrm{v}_{1}$ when computing the polynomial part of the Taylor model in the space and time variables. The Picard iteration is again performed for $\mathrm{T} \in[0, \quad 0.1]$, with initial approximations:

$$
\begin{aligned}
& U^{(0)}(T, a, b)=0.904667+1.01 a+0.1 b, \\
& V^{(0)}(T, a, b)=-0.909333+0.19 a+1.01 b+0.1 a^{2}
\end{aligned}
$$

After three iteration (and again omitting higher order terms), we obtain

$$
\begin{aligned}
\mathrm{U}^{(3)}(\mathrm{T}, \mathrm{a}, \mathrm{b})=0.904667+1.01 \mathrm{a}+0.1 \mathrm{~b} \\
-0.909333 \mathrm{~T}+0.19 \mathrm{aT}+1.01 \mathrm{bT}+ \\
0.409211 \mathrm{~T}^{2}+0.1 \mathrm{a}^{2} \mathrm{~T}+ \\
0.913713 \mathrm{aT}^{2}+0.0904667 \mathrm{bT}^{2}- \\
0.274215 \mathrm{~T}^{3} \\
\mathrm{~V}^{(3)}(\mathrm{T}, \mathrm{a}, \mathrm{b})=-0.909333+0.19 \mathrm{a}+ \\
1.01 \mathrm{~b}-0.818422 \mathrm{~T}+0.1 \mathrm{a}^{2}+ \\
1.82743 \mathrm{aT}+0.180933 \mathrm{~b}+ \\
0.180933 \mathrm{bT}-0.822644 \mathrm{~T}^{2}+ \\
1.0201 \mathrm{a}^{2} \mathrm{~T}+0.202 \mathrm{abT}+0.01 \mathrm{~b}^{2} \mathrm{~T} \\
-0.74654 \mathrm{aT}^{2}+0.82278 \mathrm{bT}^{2}+ \\
0.522429 \mathrm{~T}^{3}+ \\
\text { To compute the interval reminder }
\end{aligned}
$$
term, we must find intervals $i_{1}, j_{1}$ fulfilling the inclusions:

$$
\begin{aligned}
& u_{1}(a, b)+\int_{0}^{T}\left(v^{(3)}(s, a, b)+j_{1}\right) d s \subseteq u^{(3)}(T, a, b)+i_{1} \\
& v_{1}(a, b)+\int_{0}^{T}\left(u{ }^{(3)}(s, a, b)+i_{1}\right)^{2} d s \subseteq v^{(3)} \ldots \ldots \ldots . .(16) \\
& (T, a, b)+j_{1}
\end{aligned}
$$

For all $\mathrm{a}, \mathrm{b} \in[-0.05,0.05]$ and for all $\mathrm{T} \in[0,0.1]$ (Note that $\mathrm{i}_{0}$ and $\mathrm{j}_{0}$ are contain in $\mathrm{u}_{1}$ and $\mathrm{v}_{1}$, respectively, from (14). Suitable reminder intervals are, for example $\mathrm{i}_{1} \in[-1.12850 \mathrm{E}-4,1.65751 \mathrm{E}-4]$ 
$\mathrm{j}_{\mathrm{i}} \in[-3.31917 \mathrm{E}-4,3.24724 \mathrm{E}-4]$

Thus, the flow IVP(12) for $t \in$ $[0.1,0.2]$ is contained in the Taylor models:

$$
\begin{aligned}
& \widetilde{\mathrm{u}}_{2}(T, a, b)= \mathrm{u}^{(3)}(T, a, b)+\mathrm{i}_{1}, \\
& \widetilde{\mathrm{V}}_{2}(T, a, b)=\mathrm{v}^{(3)}(T, a, b)+\mathrm{j}_{1}, \\
& \mathrm{u}_{2}(\mathrm{~T}, \mathrm{a}, \mathrm{b})=\widetilde{\mathrm{u}}_{2}(0.1, \mathrm{a}, \mathrm{b})= 0.817551+1.03814 \mathrm{a}+0.201905 \mathrm{~b}+0.01 \mathrm{a}^{2}+\mathrm{i}_{1}, \\
& \mathrm{~V}_{2}(\mathrm{~T}, \mathrm{a}, \mathrm{b})=\widetilde{\mathrm{V}}_{2}(0.1, \mathrm{a}, \mathrm{b})=-0.835195+0.365277 \mathrm{a}+1.03632 \mathrm{~b}+0.20201 \mathrm{a}^{2} \\
& 0.0202 \mathrm{ab}+0.001 \mathrm{~b}^{2}+\mathrm{j}_{1},
\end{aligned}
$$

For larger value of $t$, the integration can be continued as in the second integration step described above. In te above example, we have used the so-called naive Taylor model integration method to illustrate the qualitative difference of interval methods and Taylor model methods for solving (IVPs). For practical computations, the naive Taylor model method is not very useful. The interval remainder terms are propagated as in the direct interval method. The inclusion (15) implies that the diameters of the interval reminder terms are nondecreasing. Often, these diameters grow exponentially, and to method break down early.

\section{Conclusion}

We have used traditional enclosure methods with Taylor model based integration. For the verified solution of IVP's for ODEs, we have shown how Taylor model methods benefit from symbolic computations. Increasd flexibility in admissible boundary curves of enclosures is an intrinsic advantage over traditional interval methods, not only for the solution of ODEs. In future research, we hope to contribute to the further development and increased use of Taylor model methods.
Where $\mathrm{a}, \mathrm{b} \in[-0.05,0.05], \mathrm{T} \in$ $[0,0.1], \mathrm{t}=\mathrm{T}+0.1$. Evaluating at $\mathrm{T}=$ 0.1 , I obtain the enclosure of the flow at $t_{2}=0.2$ (Taylor models at most 2 in the space variables):

\section{References}

1. Berz, M. and Hoffstatter, G. 1998. computation and application of Taylor polynomials with interval reminder bounds, Reliable computing. 4: PP.83-97.

2. Makino, K. and Berz, M. 2004. Suppression of the wrapping effect by Taylor model-based validated integrators. MSU Report MSUHEP 40910, Michigan state university.

3. Makino, K. and Berz, M. 1996. Remainder differential algebras and their applications, in computational differentiation: Techniques, Applications and Tools, Berz, M. Bischof, C. Coliss, G. and Griewank, A. eds SIAM, Philadelphia, PP. 63-74.

4. Makino, K. 2004. Suppression of the wrapping effect by Taylor model-based validated integrations: MSU Report MSUHEP 40910, Michigan state university.

5. Makino, K. 1998. Rigorous, analysis of nonlinear motion in particle accelerators. $\mathrm{Ph} . \mathrm{D}$ thesis, Michigan state university.

6. Nedialkov, N. S. and Jackson, K. R. 2001 A new perspective on the wrapping effect in interval method for initial value problems for 
ODEs. in Perspective of enclosure methods: Kulisch, U. Lohner, R. and Facius, A. eds, springer,wien, PP: 219-264.

7. Berz, M. and Makino, K. 1998. verified integration of ODEs and flows using differential algebraic methods on high-order Taylor methods: Reliable computing: 4: PP. 361-369.

8. Stewart, N. F. 1971. A heuristic to reduce the wrapping effect in the numerical solution of $x^{\prime}=f(t, x)$. BIT, 11: PP. 328-337.

9. Neher, M. 2001. Geometric series bounds for the local errors of Taylor methods for linear n-th order ODEs. In symbolic Algebraic methods and verification methods, Alefeld, G. Rohn, J. Rump, S. and Yamaoto, T. eds. Springer, Wien: PP. 183193.

10. Makino, K. Berz, M. and Kim, Y. 2004. Range bounding with Taylor models. Some case studies, WSEAS Transactions on Mathematics, 3: PP. 137-145.

11. Berz, M. 1997. From Taylor series to Taylor models, in AIP conference Proceedings 405: PP. 1-23. 
نموذج مبسط لطريقة تبلر للتكامل

شوقي عبد المطلب عباس"

* قسم الرياضيات/ كلية العلوم/ جامعة بغداد

الخلاصة

طر ائق الفترات لتحقيق التكامل للمسائل ذات القيم الابتدائية (IVPS) للمعادلات التفاضلية الاعتياديـة

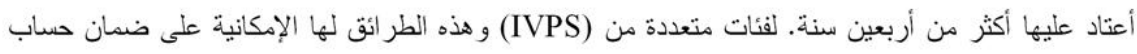

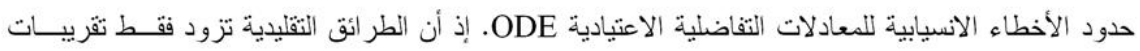

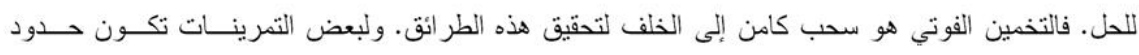

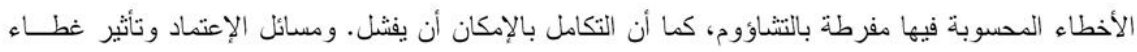

التظليف تكون عملياً مصادر التخمين الفوقي لحساب الفترات.

وطور العالم (Berz) مع مساعديه أنموذجاً لطريقة تيلر التي نشرت حساب الفتـرات ذي الحسـابات

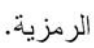

و الأخيرة أداة فعالة لتقليص كل من مسألة الاعتماد وتأثير تغليف الغطاء. بيناء أنموذج طريقــة تيلــر

التي تظهر بشكل علي ملائمة التكاملات التفاضلية الاعتيادية غير الخطية (ODEs).

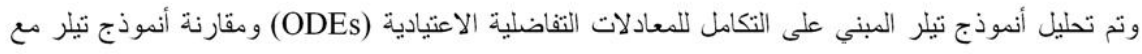

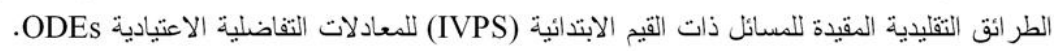

كلمات مفتاحية: موديل طريقة نبلر ، نحقيق التكامل، IVPs،ODEs. 